\title{
19. New Evidence for Interplanetary Boulders?
}

\author{
Zdenek Sekanina \\ Smithsonian Astrophysical Observatory \\ Cambridge, Massachusetts
}

\begin{abstract}
The present paper critically discusses the method of detection, the magnitude and the rate of occurrence of sudden disturbances in the motions of some short-period comets. The disturbances have recently been suggested as potential indicators of collisions between the comets and interplanetary boulders-minor objects whose existence was predicted by $M$. Harwit in 196\%. The character of explosive phenomena, caused by an impact of such a boulder on a comet's nuclear surface, depends significantly on the surface texture of the target body. To advance our understanding of the impact mechanism, a method is suggested which would supply a good deal of the missing information about the structure and optical properties of nuclear surfaces from precise photometric observations of cometary nuclei at large solar distances.
\end{abstract}

\section{THE IMPACT HYPOTHESIS}

$\mathrm{R}$ ECENT EXTENSIVE DYNAMICAL STUDIES of a number of short-period comets by Marsden (1969, 1970), by Yeomans (1972), and by Marsden and Sekanina (1971) resulted in a discovery of easily detectable disturbances in the motions of the comets we call 'erratic': P/Biela, $\mathrm{P} /$ Brorsen, $\mathrm{P} /$ Giacobini-Zinner, $\mathrm{P} /$ Perrine-Mrkos, $\mathrm{P} /$ Schaumasse, possibly also $\mathbf{P} /$ Forbes and $\mathrm{P} /$ Honda-Mrkos-Pajdušáková. The disturbances differ from the regular nongravitational effects and seem to take form of sudden impulses of about $1 \mathrm{~m} / \mathrm{s}$, perhaps preferably at larger solar distances. Their interpretations in terms of processes stimulated by internal cometary sources of energy have been discarded on various grounds. In contrast, hypervelocity impacts of small objects seem to be consistent with empirical evidence. The existence of interplanetary boulders has been predicted by Harwit (1967), of spatial density as high as $10^{-18} \mathrm{~g} / \mathrm{cm}^{3}$. Application of the mechanism of crater formation at hypervelocity impacts suggests that the observed disturbances can be generated by collisions of the boulders with low density comet nuclei, if the comet-to-boulder mass ratio is about $10^{6}$. As a result of such an impact the comet would lose as much as $\mathbf{1 0}$ percent of its mass. Repeated impacts can easily result in a splitting of the nucleus, or its complete disintegration in a relatively short period of time. With Harwit's space mass density of boulders the proposed hypothesis predicts an average rate of some five impacts per 100 revolutions for a comet $1 \mathrm{~km}$ in diameter. To produce an impulse of $1 \mathrm{~m} / \mathrm{s}$ the average boulder should be $10^{8} \mathrm{~g}$ in mass, or 3 to $10 \mathrm{~m}$ in diameter, depending on its mass density; the comet would be $10^{14} \mathrm{~g}$ in mass, and $0.2 \mathrm{~g} / \mathrm{cm}^{3}$ in density. Such a nucleus can be composed of snows mixed with highly porous dust grains. Impacts of the same boulders would not measurably affect motions of the comets with heavy compact cores like P/Encke.

These have been some of the main conclusions formulated by Marsden and Sekanina (1971) from their extensive study of the motions of the 
'erratic' comets. In the present paper we discuss numerical values of some of the fundamental constants of the problem in greater detail. We will refer to the above paper as to Paper 1 .

\section{RADIAL AND TRANSVERSE COMPONENTS OF THE NONGRAVITATIONAL EFFECTS IN THE DAILY MEAN MOTION}

An acceleration, the radial component of which is $Z_{1}$ (positive outward from the Sun) and transverse component $Z_{2}$ (perpendicular to $Z_{1}$ in the orbit plane, positive in the direction of motion), applied at a solar distance $r$ generates an instantaneous rate of change in the daily mean motion

$$
\dot{\mu}=-\frac{3 e \sin v}{a\left(1-e^{2}\right)^{1 / 2}} Z_{1}-\frac{3\left(1-e^{2}\right)^{1 / 2}}{r} Z_{2}
$$

where $v$ is the true anomaly, $a$ and $e$ are the semimajor axis and eccentricity of the orbit. We accept that the nongravitational acceleration varies with the solar distance $r$,

$$
\begin{aligned}
Z_{i} & =A_{i} f(r) \\
f(1 \mathrm{AU}) & =1 \\
i & =1,2,3
\end{aligned}
$$

where $A_{i}$ are the acceleration components at $1 \mathrm{AU}$ in units of the solar gravitational acceleration at 1 AU. Upon integrating over a revolution period, and writing

$$
I_{j}=\int_{-\pi}^{\pi} f(r) r^{j} d v
$$

we obtain the change in the daily mean motion per revolution:

$$
\Delta \mu=-3 A_{2} k I_{1} a^{-1 / 2}
$$

There is no contribution from the periodic variations in the radial component of the acceleration. However, there is a secular effect from $Z_{1}$ because of its contribution to the "effective" gravitational constant. On the one hand we have

$$
\Delta\left(\int_{0}^{P} \frac{k^{2}}{r^{2}} d t\right)=2 \pi p^{-1 / 2} \Delta k=2 \pi p^{-1 / 2} a^{3 / 2}(\Delta \mu)_{r}
$$

where $p=a\left(1-e^{2}\right)$ and $(\Delta \mu)_{r}$ is the change, per revolution, in the daily mean motion due to the change in the Gaussian constant $k$. On the other hand, from equation (2),

$$
\Delta\left(\int_{0}^{P} \frac{k^{2}}{r^{2}} d t\right)=-A_{1} k I_{2} p^{-1 / 2}
$$

so that

$$
(\Delta \mu)_{r}=-(2 \pi)^{-1} A_{1} k I_{2} a^{-3 / 2}
$$

It is convenient to convert $\Delta \mu$ of equation (4) to $\Delta T_{2}$ and $(\Delta \mu)_{r}$ of equation (7) to $\Delta T_{1}$, the effective rates of delay $\left(\Delta T_{i}>0\right)$ or advance $\left(\Delta T_{i}<0\right)$ in the perihelion passage per revolution due to, respectively, the transverse and radial components of the acceleration of equation (2). Expressing $\Delta T_{i}$ in days per revolution per revolution we find

$$
\begin{aligned}
& \Delta T_{1}=58 A_{1} I_{2} a^{3 / 2} \\
& \Delta T_{2}=1096 A_{2} I_{1} a^{5 / 2}
\end{aligned}
$$

The integrals $I_{1}$ and $I_{2}$ are of the same order of magnitude. For a typical short-period comet ratio $\Delta T_{2} / \Delta T_{1}$ is about 10 , even when the radial component is almost an order of magnitude larger than the transverse component.

\section{DYNAMICAL DISTURBANCES}

The computer programs used by the authors, mentioned earlier for calculating the orbital elements and nongravitational parameters from comet observations, are designed to search for smoothly, continuously varying deviations from the gravitational law. If a disturbance is detected by the program in a comet's motion, contradicting the above assumption, the integration procedure does not necessarily fail. What does happen depends much on the number of the comet's apparitions linked. A solution may be found, which gives quite an acceptable distribution of residuals, but the nongravitational parameters are inconsistent with those computed from the comet's adjacent apparitions not including the dynamical anomaly. Figure 1 shows an example of such a forced solution. The regular nongravitational effects shape the continuous background SABEFZ. Between $t_{B}$ and $t_{E}$ a disturbance BCTDE is superposed on the quiescent phase $\mathrm{BE}$. If an attempt is made to link apparitions between $t_{A}$ and $t_{z}$, and the nongravitational effects are allowed to vary exponentially with time,

$$
A \sim \exp (-B t)
$$

the empirical fit yields the curve $A^{\prime} C D F Z^{\prime}$ such 


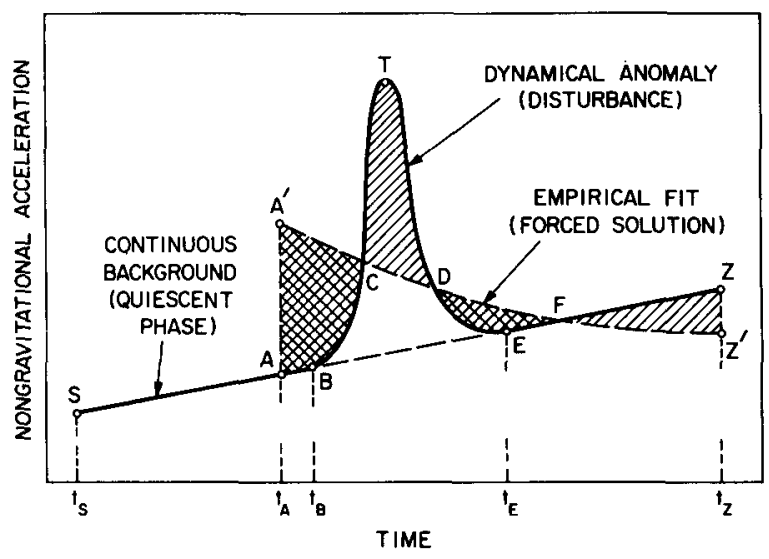

Figure 1.-Sudden dynamical anomaly, or disturbance, interferes with continuous, quiescent-phase nongravitational effects in a comet's motion. The disturbance can be detected by means of a 'forced' solution: the cross-shaded areas compensate the one-way shaded areas.

that

$$
\begin{aligned}
& \operatorname{area}\left(\mathrm{AA}^{\prime} \mathrm{CBA}\right)+\operatorname{area}(\mathrm{DFED}) \\
& =\operatorname{area}(\mathrm{CTDC})+\operatorname{area}\left(\mathrm{FZZ}^{\prime} \mathrm{F}\right)
\end{aligned}
$$

The coefficient $B$ of the secular variationsnegative in the quiescent phase in figure 1suddenly becomes positive due to the disturbance.

Thus, figure 1 is a very obvious demonstration that whenever a disturbance is involved, $B$ comes out fictitious. It is easy to understand that the sign and magnitude of $B$ depends not only on the disturbance-to-background ratio, but also on the selected span of time. For example, $B$ would be strongly negative, if we tried to link apparitions between $t_{S}$ and $t_{E}$. Moreover, the forces generating the quiescent-phase and disturbance effects may work in opposite directions, and we may fail to find a satisfactory solution of the form of equation (9) and must accept another empirical form. If only three apparitions are linked it is always possible to find a satisfactory solution with constant $A$. Figure 1 corresponding to this case would have a staircase shape, and the general rule, equation (10), would again be in power. In practice, however, the validity of equation (10) is only approximate. The reason comes from the difference between the real orbit (with the unknown profile of the disturbance) and the fictitious orbit found by the forced continuous solution. The differential perturbations, predominantly due to
Jupiter, along the two orbits should be taken into account in equation (10). In practice, the perturbations are very small unless the comet makes a close approach to Jupiter during the critical period of time. Unfortunately, these encounters are fairly frequent and often limit our results in accuracy.

\section{SUDDEN IMPULSES}

We do not--and practically cannot-have direct evidence of the character of the dynamical disturbances affecting the 'erratic' comets. We guess that they take form of discrete discontinuities (see Paper 1), because so far it has always been found that observations from only the minimum number of apparitions, necessary for the leastsquares procedure to work, can satisfactorily be fitted whenever a disturbance is involved. Outside that span the forced solution completely fails. Typically, there are long intervals of quiescent phase before such a disturbance ( $\mathrm{P} /$ GiacobiniZinner), or after it (P/Biela), or both before and after ( $\mathrm{P} /$ Schaumasse).

If we take the disturbance in the form of a sudden impulse $\left(t_{B} \rightarrow t_{E}\right.$ in fig. 1) and are able to estimate the quiescent-phase background, we can determine the impulsive increment in the orbital velocity, $\Delta V_{v}$, associated with the discontinuity, from the difference between the disturbed and quiescent nongravitational parameters. The impulse corresponds to the area BCTDEB in figure 1.

The component of the nongravitational acceleration along the orbital velocity vector, $Z_{v}$, is given by

$$
Z_{v}=\left(Z_{1} V_{1}+Z_{2} V_{2}\right) V^{-1}
$$

where $Z_{1}, Z_{2}$ are identical with those of equation (2), $V$ is the orbital velocity, $V_{1}$ and $V_{2}$ its radial and transverse components respectively. Integrating over the revolution period, we have from equation (11)

$$
\psi=\int_{0}^{P} Z_{v} d t=\kappa A_{2} \int_{-\pi}^{\pi} f(r) r\left\{\frac{2}{r}-\frac{1}{a}\right\}^{-1 / 2} d v
$$

where $v$ is the true anomaly; $\kappa=29.8 \times 10^{3}$, if $\psi$ is to be given in meters per second. The expression, equation (12), is independent of $Z_{1}$ for the reasons discussed above.

Let $\psi_{\text {quiese }}$ and $\psi_{\text {distrb }}$ be $\psi$ for the quiescent phase 
and the disturbed period, respectively. The impulsive increment in the orbital velocity associated with the disturbance is then

$$
\begin{aligned}
\Delta V_{v} & =\left(\psi_{\text {distrb }}-\psi_{\text {quiesc }}\right) \nu \\
& +(\text { effect of differential perturbations })
\end{aligned}
$$

where $\nu$ is the number of revolutions covered by the forced fit. The positive $\Delta V_{v}$ means the comet is effectively decelerated, the negative means accelerated. If $A_{2}$ has been allowed to be subject to secular variations, its effective value during the period of time covered by the forced fit must be used in equation (12).

The total impulsive velocity, $\Delta V$, associated with the disturbance cannot be derived from its component along the orbital velocity vector, because the angle between the impulse and the orbit tangent is not known. Assuming that the discontinuity in motion is due to a collision with a small object moving in a circular orbit around the Sun in the comet's orbital plane, we have derived in Paper 1 the following formula for the mean quadratic relative velocity between the two colliding bodies, averaged by integration over a revolution period:

$$
\begin{aligned}
w & =\left(\overline{w^{2}}\right)^{1 / 2} \\
& =\kappa a^{-1 / 2}\left[2-\frac{4}{\pi}(1-e)^{1 / 2} K\left\{(2 e)^{1 / 2}(1+e)^{-1 / 2}\right\}\right]^{1 / 2}
\end{aligned}
$$

where $K\{m\}$ is the complete elliptic integral of the first kind with modulus $m$. Similarly we can calculate the mean quadratic component of the relative collision velocity in the direction of the

Table 1.-Ratio $\overline{\Delta V} / \Delta \mathrm{V}_{v}$ vs Eccentricity, $\mathrm{e}$

\begin{tabular}{c|c}
\hline$e$ & $\overline{\Delta V} / \Delta V_{v}$ \\
\hline 0.1 & \\
0.2 & 2.24 \\
0.3 & 2.22 \\
0.4 & 2.19 \\
0.5 & 2.15 \\
0.6 & 2.10 \\
0.7 & 2.04 \\
0.8 & 1.96 \\
0.9 & 1.87 \\
& 1.75 \\
\hline
\end{tabular}

comet's motion:

$$
\begin{aligned}
w_{v}=\left(\overline{w_{v}}\right)^{1 / 2}= & \kappa a^{-1 / 2}\left[1+\left(1-e^{2}\right)^{1 / 2}-\frac{4}{\pi}(1-e)^{1 / 2}\right. \\
& \left.\cdot K\left\{(2 e)^{1 / 2}(1+e)^{-1 / 2}\right\}\right]^{1 / 2},
\end{aligned}
$$

Relating the direction of the impulsive velocity to that of the relative collision velocity we now can find $\Delta V$ averaged over a revolution period:

$$
\begin{aligned}
\overline{\Delta V} & =\Delta V_{v} \\
& \times\left[1-\frac{1-\left(1-e^{2}\right)^{1 / 2}}{2-\frac{4}{\pi}(1-e)^{1 / 2} K\left\{(2 e)^{1 / 2}(1+e)^{-1 / 2}\right\}}\right]^{-1 / 2}
\end{aligned}
$$

The ratio $\overline{\Delta V} / \Delta V_{v}$ is listed in table 1 as a function of the eccentricity.

\section{IMPULSIVE VELOCITIES ASSOCIATED WITH THE DISTURBANCES OBSERVED IN MOTIONS OF THE 'ERRATIC' COMETS}

The method described in the preceding section can give a reasonable estimate of the impulsive velocity, particularly if there is no close approach to Jupiter involved. Table 2 lists $\Delta V_{v}$ and $\overline{\Delta V}$ obtained in this way for the 'erratic' comets and compares them with the values derived by Marsden for $\mathrm{P} /$ Schaumasse, $\mathrm{P} /$ Perrine-Mrkos and $\mathrm{P} /$ Biela, and by Yeomans for $\mathrm{P} /$ GiacobiniZinner, who have used a different approach. These authors have computed what we call $\Delta V_{v}$ from the difference between the observed time of perihelion passage and that extrapolated from a quiescent phase.

The fundamental difference between the two methods is that the one we suggest tends to smooth the disturbance out and represents therefore a lower limit of the most probable impulsive velocity. On the other hand, the method applied by Marsden and by Yeomans extrapolates, and therefore tends to exaggerate the effect of the disturbance. Indeed, table 2 clearly shows that our values of the impulsive velocity are systematically smaller. In any case, the table suggests that $1 \mathrm{~m} / \mathrm{s}$, which was accepted in Paper 1 for the 
TABLE 2.-'Erratic' Comets: Impulsive Velocities Assaciated With Dynamical Disturbances

\begin{tabular}{|c|c|c|c|c|c|c|}
\hline \multirow{2}{*}{ Comet $^{\mathrm{a}}$} & \multirow{2}{*}{$\begin{array}{l}\text { Disturbance } \\
\text { between- }\end{array}$} & \multirow{2}{*}{$\begin{array}{c}\Delta V_{v} \\
\text { from eq. } \\
(13)(\mathrm{m} / \mathrm{s})\end{array}$} & \multirow{2}{*}{$\begin{array}{c}\overline{\Delta V} \\
\text { from eq. } \\
(16)(\mathrm{m} / \mathrm{s})\end{array}$} & \multirow{2}{*}{$\begin{array}{c}\Delta V_{v} \\
\text { derived } \\
\text { otherwise } \\
(\mathrm{m} / \mathrm{s})\end{array}$} & \multicolumn{2}{|c|}{$\begin{array}{l}\text { Comet's mean motion } \\
\text { effectively }\end{array}$} \\
\hline & & & & & $\begin{array}{l}\text { In quiescent } \\
\text { phase }\end{array}$ & $\begin{array}{c}\text { By } \\
\text { disturbance }\end{array}$ \\
\hline \multirow[t]{2}{*}{$\mathrm{P} /$ Biela } & $1772 / 1805$ & 0.8 & 1.5 & & $A$ & $D$ \\
\hline & d $1842 / 43$ & 0.1 & 0.2 & ${ }^{\ominus} 1 \quad M_{2}$ & $A$ & $D$ \\
\hline \multirow[t]{2}{*}{ P/Brorsen } & f $1846 / 73$ & $(0.3)$ & $(0.5)$ & & $A$ & $?$ \\
\hline & $1873 / 79$ & 1.6 & 3.0 & & $A$ & $D$ \\
\hline $\mathrm{P} /$ Schaumasse & $1927 / 43$ & 0.2 & 0.5 & $1.9 M_{1}$ & $A$ & $A$ \\
\hline $\mathrm{P} /$ Perrine-Mrkos & $1955 / 68$ & 1.5 & 3.0 & $3.5 M_{1}$ & $A$ & $A$ \\
\hline $\mathrm{P} /$ Giacobini-Zinner & $1959 / 65$ & 0.6 & 1.1 & $1.4 Y$ & $D$ & $D$ \\
\hline
\end{tabular}

" Data on $\mathrm{P} / \mathrm{Honda-Mrkos-Pajdu}$ áková and $\mathrm{P} /$ Forbes are inconclusive.

b $M_{1}=$ Marsden (1970); $M_{2}=$ Marsden (1971) [also in Marsden, Sekanina (1971)]; $Y=$ Yeomans (1971).

- $A=$ accelerated $D=$ decelerated.

d Satellite nucleus at splitting.

- Velocity of separation essentially in sunward direction.

f Impulsive velocity rather uncertain.

typical impulsive velocity, seems indeed to be a representative value for the 'erratic' comets.

\section{RATE OF DISTURBANCES}

The rate of dynamical disturbances is another critical quantity for the impact hypothesis. Table 3 lists the number of observed disturbances, $\mathfrak{n}$, for the 'erratic' comets; the length, $\ell$, of their trajectories swept out between the first and last observed apparitions of each of the comets; and the disturbance rate, $\Gamma$, defined as the number of disturbances per $100 \mathrm{AU}$ :

$$
\Gamma=100 \Re / \ell
$$

For the known 'erratic' comets the average observed rate is about one disturbance per $100 \mathrm{AU}$ (see column 6 of table 3 ), a value about four times as high as the one predicted from the impact hypothesis in Paper 1. However, it is easy to show that the data of table 3 are strongly affected by observational selection.

The number of boulder impacts on a cometary nucleus is statistically proportional to the volume of space swept out by the comet. The volume is given as a product of the comet's collisional crosssection and the length of its trajectory. Ideally,
$\Re$ should depend linearly on $\ell$ in figure 2 . However, since we deal with observed lengths of trajectories that are very short compared to the rate of presumed impacts, statistical dispersion is significant and the observed disturbance rates for individual comets differ widely from each other. We identify only the best observed 'erratic' comets. It is therefore logical that the upper left corner of figure 2 , the area of the highest disturbance rates, is populated most, whereas the strongest bias takes place along $\mathfrak{N}=\mathbf{0}$. Observational selection is also responsible for a factor of three between the mean least-square rate $\mathfrak{N} / \ell$ (dot-and-dashed line) and the differential rate $d \mathscr{N} / d \ell$ (dashed line). It is the latter that should more properly match the unbiased disturbance rate. Indeed, the rate predicted from the impact hypothesis (solid line) agrees with the slope $d \Re / d \ell$.

For the above reasons it is convenient to write the number of the 'erratic' comets with disturbance rates between $\Gamma$ and $\Gamma+d \Gamma$ in the form

$$
d N_{c}(\Gamma)=N_{c} \phi(\Gamma) d \Gamma, \int_{0}^{\infty} \phi(\Gamma) d \Gamma=1
$$

and to see how the total number, $N_{c}$, of the 'erratic' comets among known short-period comets and 
TABLE 3.-'Erratic' Comets: Observed Rates of Dynamical Disturbances

\begin{tabular}{l|l|c|c|c|c}
\hline \multicolumn{1}{c|}{ Comet } & $\begin{array}{c}\text { First/last } \\
\text { apparition }\end{array}$ & $\begin{array}{c}\text { Number of } \\
\text { revolutions } \\
\text { covered }\end{array}$ & $\begin{array}{c}\text { Length of } \\
\text { swept-out } \\
\text { trajectory } \\
\text { (AU) }\end{array}$ & $\begin{array}{c}\text { Number of } \\
\text { detected } \\
\text { disturbances }\end{array}$ & $\begin{array}{c}\text { Disturbance } \\
\text { rate } \\
\text { (per 100 AU) }\end{array}$ \\
\hline P/Biela & $1772 / 1852$ & 12 & 226 & 2 & \\
P/Brorsen & $1846 / 79$ & 6 & 95 & 2 & 2.10 \\
P/Schaumasse & $1911 / 60$ & 6 & 131 & 1 & 0.76 \\
P/Perrine-Mrkos & $1896 / 1968$ & 11 & 210 & 1 & 0.48 \\
P/Giacobini-Zinner & $1900 / 66$ & 10 & 187 & 1 & 0.53 \\
P/Honda-Mrkos-Pajduśáková & $1948 / 69$ & 4 & 61 & $1 ?$ & 1.23 \\
P/Forbes & $1929 / 61$ & 5 & 100 & $1 ?$ & 0.75 \\
\hline
\end{tabular}

a The disturbance rate of this comet is weighted by a factor of $3 / 4$ to allow for the uncertainty as to whether the disturbance indeed occurred.

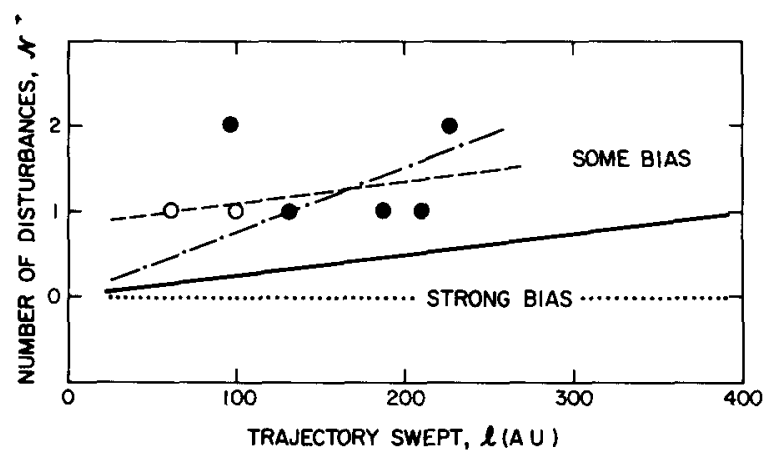

Figure 2.-Observed dynamical disturbances. Solid circles: definite 'erratic' comets. Open circles: probable 'erratic' comets. Solid line: disturbance rate predicted from the impact hypothesis (see Paper 1). Dot-anddashed line: least-square solution to the mean observed disturbance rate, $\mathfrak{N} / \ell$. Dashed line: leastsquare solution to the mean-differential disturbance rate, $d \mathfrak{N} / d \ell$.

the characteristic disturbance rate, $\Gamma_{c}$, given by

$$
\int_{0}^{\Gamma_{e}} \phi(\Gamma) d \Gamma=\int_{\Gamma_{c}}^{\infty} \phi(\Gamma) d \Gamma
$$

depend on the choice of the distribution function $\phi(\Gamma)$. The number of comets with disturbance rates higher than $\Gamma$,

$$
N_{c}+(\Gamma)=N_{c} \int_{\Gamma}^{\infty} \phi(\gamma) d \gamma
$$

proves the most useful quantity for practical trials, because our statistics of disturbances is relatively complete for very high values of $\Gamma$.

Approximating $\phi(\Gamma)$ first by the Maxwellian velocity distribution function, with $\Gamma_{m}$ being the most frequent $\Gamma$,

$\phi(\Gamma) d \Gamma=4 \pi^{-1 / 2} \Gamma^{2} \Gamma_{m}^{-3} \exp \left[-\left(\Gamma / \Gamma_{m}\right)^{2}\right] d \Gamma$

we find

$$
\begin{aligned}
N_{c}+(\Gamma)= & N_{c}\left\{2 \pi^{-1 / 2} \Gamma \Gamma_{m}^{-1}\right. \\
& \left.\times \exp \left[-\left(\Gamma / \Gamma_{m}\right)^{2}\right]+\operatorname{erfc}\left(\Gamma / \Gamma_{m}\right)\right\}
\end{aligned}
$$

where

$$
\operatorname{erfc}(x)=1-2 \pi^{-1 / 2} \int_{0}^{x} \exp \left(-x^{2}\right) d x
$$

The characteristic rate is

$$
\Gamma_{c}=1.087 \Gamma_{m}
$$

The fit to the empirical data is, however, unsatisfactory.

Alternatively, we can assume that $\phi(\Gamma)$ has the form of a two-dimensional Maxwellian velocity distribution. This assumption seems to be more plausible in view of presumably low obliquities between orbital planes of the short-period comets and interplanetary boulders. Then

$$
\begin{aligned}
\phi(\Gamma) d \Gamma & =\Gamma \Gamma_{m}{ }^{-2} \exp \left[-\Gamma^{2} / 2 \Gamma_{m}^{2}\right] d \Gamma \\
N_{c}+(\Gamma) & =N_{c} \exp \left[-\Gamma^{2} / 2 \Gamma_{m}^{2}\right]
\end{aligned}
$$

and

$$
\Gamma_{c}=\Gamma_{m}\left(\log _{e} 4\right)^{1 / 2}
$$

There is an improvement upon equation (22) in matching the data of table 3 , but the fit is still poor for $N_{c}^{+}<3$. 
Let us next accept an exponentially decreasing distribution

$$
\phi(\Gamma) d \Gamma=\beta^{-1} \exp [-\Gamma / \beta] d \Gamma
$$

so that

$$
N_{c}+(\Gamma)=N_{c} \exp [-\Gamma / \beta]
$$

and

$$
\Gamma_{c}=\beta \cdot \log _{e} 2
$$

The fit is now good except for $N_{c}{ }^{+}=1$.

Finally, if we take

$$
\begin{aligned}
\phi(\Gamma) d \Gamma & =(2 \zeta)^{-1} \Gamma^{-1 / 2} \exp \left[-\Gamma^{1 / 2} / \zeta\right] d \Gamma \\
N_{c}+(\Gamma) & =N_{c} \exp \left[-\Gamma^{1 / 2} / \zeta\right] \\
\Gamma_{c} & =\left(\zeta \cdot \log _{e} 2\right)^{2}
\end{aligned}
$$

we get a very good fit to all the seven data.

Unfortunately, the testing data are too scanty to resolve the ambiguity in $\phi(\Gamma)$ unequivocally, and the best fit does not necessarily mean the best solution. Indeed, a fit at least as good as that by equation (32) is obtained from

$$
N_{c}^{+}(\Gamma)=2.7 \Gamma^{-1.4}
$$

which gives no prediction for $N_{c}$ whatsoever.

Table 4 lists the characteristic rate $\Gamma_{c}$ and the extrapolated $N_{c}$ for the four applied $\phi(\Gamma)$. The dependence of the two parameters on the character of $\phi(\Gamma)$ is significant. The mean rate of disturbances computed in Paper 1 from equations of the impact hypothesis, certain physical assumptions and dynamical evidence comes out 0.25 per $100 \mathrm{AU}$ (solid line in fig. 3 of paper 1), which is in order-of-magnitude agreement with the data of table 4 .

\section{PHASE EFFECT AND ALBEDO OF COMETARY NUCLEI}

The dimensions and mass of an average 'erratic' comet are important for the impact hypothesis for two reasons:

(1) The impact rate is proportional to the collisional cross-section of the comet's nucleus.

(2) If the impulsive velocity is known, the mass of the nucleus determines the magnitude of the impulse exerted by a boulder impact, which equals the momentum gained by the material expelled from the nucleus. The momentum, in turn, determines the mass of the boulder.

An upper limit for a cometary radius can be derived from dynamical considerations of a cometary splitting. For $\mathrm{P} /$ Biela (classed as an 'erratic' comet) the requirement that the separation velocity be higher than the velocity of escape from the surface of the nucleus of radius $R$ and mean density $\rho$ gives a condition

$$
R \rho^{1 / 2}<1.3 \mathrm{~km}\left(\mathrm{~g} \mathrm{~cm}^{-3}\right)^{1 / 2}
$$

For $\rho \simeq 0.2 \mathrm{~g} \mathrm{~cm}^{-3}$ we find $R<3 \mathrm{~km}$. If we require that the separation velocity exceeds the escape velocity from the sphere of action of the nucleus we must use another formula (Sekanina, 1968) and get for P/Biela

$$
R \rho^{1 / 3}<12 \mathrm{~km}\left(\mathrm{~g} \mathrm{~cm}^{-3}\right)^{1 / 3}
$$

or $R<20 \mathrm{~km}$ for a low-density snowball. These estimates are too crude to be used for the calculation of an 'erratic' comet's mass.

The photometry of faint cometary images at large solar distances appears to be more fruitful

'TABLE 4.--Erratic' Comets: Total Number and Characteristic Disturbance Rate as a Function of the Disturbance Frequency Distribution

\begin{tabular}{l|c|c|c}
\hline \multicolumn{1}{c|}{ Distribution $\phi(\Gamma)$ assumed } & $\begin{array}{c}\text { Characteristic disturbance } \\
\text { rate } \Gamma_{c} \\
\text { (per } 100 \mathrm{AU})\end{array}$ & $\begin{array}{c}\text { Total of expected } \\
\text { 'erratic' comets } N_{c}\end{array}$ & Data fit \\
\hline Three dimensional Maxwellian & 0.77 & 8 & $\begin{array}{c}\text { poor } \\
\text { Two dimensional Maxwellian }\end{array}$ \\
$\begin{array}{l}\text { Damped exponential in } \Gamma \\
\text { Damped exponential in } \sqrt{\Gamma}\end{array}$ & 0.70 & 10 & good for $N_{c}^{+}>2$ \\
good for $N_{c}^{+}>1$ \\
$\operatorname{good}$
\end{tabular}


for analyzing the dimensions of cometary nuclei. However, the practical solution of the problem is very delicate not only because of the obvious observational difficulties (very faint images; coma contamination must be reduced as much as possible), but also because of an ambiguity in interpretation.

Disregarding the sources of periodic or quasiperiodic variations in brightness (such as the shape of the nucleus), we shall deal with the geometrical albedo and phase law, the two quantities that are determined by the optical properties of the nuclear surface and enter the reduction photometric formula, from which the nuclear diameter is computed.

There is no chance to obtain direct information about the nuclear reflectivity from ground-based observations. However, it might be possible to determine the phase law from very accurate photometric observations.

The two candidates for the surface texture to be considered in reference to the impact hypothesis proposed in Paper 1, namely snow of $\mathrm{H}_{2} \mathrm{O}$ and asteroid-like compact but porous material, differ considerably from each other in both the reflectivity and the phase variations (Sekanina, 1971). A smooth surface of unpacked $\mathrm{H}_{2} \mathrm{O}$ snow has a geometrical albedo 0.5 , a phase coefficient $\beta \simeq 0.002$ mag $\mathrm{deg}^{-1}$ for small phase angles and generally resembles a Lambert surface (Veverka, 1970). On the other hand, a typical geometrical albedo for asteroids is about 0.15 , and the phase coefficient is characteristically $\beta \simeq 0.03 \mathrm{mag} \mathrm{deg}^{-1}$. For Icarus, this law is still correct at phase angles as large as $100^{\circ}$ (Gehrels et al., 1970).

Incorporation of the significant phase effect into the photometric formula brings the absolute brightness up by $0.5^{\mathrm{m}}$ at phase angle $18^{\circ}$, by $1^{\mathrm{m}}$ at $42^{\circ}$, and by more than $1^{\mathrm{m}}$ everywhere between $42^{\circ}$ and $127^{\circ}$, as compared to the Lambert law. Because a strong phase effect also implies a lower albedo, hence a larger cross section, an average asteroid-like nucleus would be larger in diameter than a snow covered nucleus by a factor $2.5,3$ and 3.5, while both nuclei have equal apparent magnitudes under equal geometrical conditions at phase angles $25^{\circ}, 48^{\circ}$ and $75^{\circ}$, respectively.

Obviously, the discrimination of cometary nuclei by the phase effect can significantly improve the accuracy of the photometric determinations of cometary radii, and thus bring down the uncertainty in the mass of individual comets by at least one order of magnitude.

Unfortunately, nuclear magnitudes of the quality required by the suggested phase-discrimination method are not available. To illustrate the difficulties encountered in an attempt to detect phase variations in published sets of magnitudes we have compiled table 5 from the homogeneous series of photographic magnitudes of comets at large solar distances, obtained by Roemer and her collaborators (Roemer, 1965, 1967, 1968; Roemer and Lloyd, 1966; Roemer et al., 1966). The table lists a sample of more extensively observed comets of the two types considered in Paper 1 ('erratic' and core-mantle). The type identification based on the dynamical evidence is given in column 2 , the degree of consistency with the phase-law evidence is commented on in the last column. Two phase laws have been tested in terms of the dispersion in the absolute magnitude, $H_{0}: \beta=0.00 \mathrm{mag} \mathrm{deg}^{-1}$, an approximation good for a smooth snow surface and therefore presumably suitable for the 'erratic'

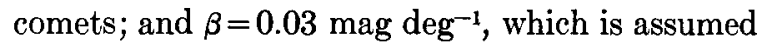
to work reasonably well for the core comets. The absolute magnitudes have been computed from apparent magnitudes, applying the inverse square reduction law. Only magnitudes from solar distances larger than 1.2 AU have been made use of, so that phase angles have been conveniently kept within $50^{\circ}$. Table 5 reveals that except for $\mathrm{P} /$ Arend-Rigaux and perhaps $\mathrm{P} /$ Encke, the dispersion in $H_{0}$ is rather high and the results therefore inconclusive. A particularly bothering trouble is a systematic difference between the absolute magnitudes at successive apparitions of the same comet. This effect is most noticeable in the case of $\mathrm{P} /$ Giacobini-Zinner.

For the sake of comparison we have also calculated the quantities of columns 3 to 5 of table 5 for two minor planets of the Apollo type. For Adonis, using photographic magnitudes by six observers, we obtain respectively $+0.7^{\mathrm{m}}, \pm 0.47^{\mathrm{m}}$ and $\pm 0.39^{\mathrm{m}}$ (the minimum being $\pm 0.36^{\mathrm{m}}$ for $\beta=0.06 \mathrm{mag} \mathrm{deg}^{-1}$ ). For $1960 \mathrm{UA}$, Object Giclas, we have used photographic magnitudes by Roemer (1965) and photoelectric $B$ magnitudes (reduced to the photographic system) by Rakos (1960), and found, respectively, $+0.5^{\mathrm{m}}, \pm 0.37^{\mathrm{m}}$ and 
TABLe 5.-Phase effect $[\beta=$ Phase Coefficient $(\mathrm{mag} / \mathrm{deg})]$

\begin{tabular}{|c|c|c|c|c|c|}
\hline \multirow{2}{*}{ Comet } & \multirow{2}{*}{$\begin{array}{c}\text { Interpretation } \\
\text { of dynamical } \\
\text { evidence }^{\mathbf{a}}\end{array}$} & \multirow{2}{*}{$\begin{array}{l}\text { Average effect of phase } \\
\text { in absolute magnitude: } \\
\qquad \begin{array}{c}H_{0}(\beta=0.00)- \\
H_{0}(\beta=0.03) \\
\text { (mag) }\end{array}\end{array}$} & \multicolumn{2}{|c|}{ Dispersion in $H_{0}$} & \multirow{2}{*}{$\begin{array}{l}\text { Agreement: } \\
\text { phase effect } \\
\text { vs dynamics }\end{array}$} \\
\hline & & & $\begin{array}{c}\beta=0.00 \\
(\mathrm{mag})\end{array}$ & $\begin{array}{c}\beta=0.03 \\
(\mathrm{mag})\end{array}$ & \\
\hline P/Encke & core & +0.5 & \pm 0.47 & \pm 0.29 & yes \\
\hline P/Giacobini-Zinner & erratic & b 0.8 & $\circ 0.70$ & ${ }^{\circ} 0.75$ & yes? \\
\hline $\mathrm{P} /$ Schaumasse & erratic & 0.8 & 0.41 & 0.54 & yes \\
\hline P/Tempel 2 & core & 0.7 & 0.45 & 0.60 & no \\
\hline $\mathrm{P} /$ Arend-Rigaux & core & 0.6 & 0.39 & 0.15 & yes \\
\hline $\mathrm{P} /$ Forbes & erratic? & 0.7 & 0.76 & 0.65 & no? \\
\hline P/Schwassmann-Wachmann 2 & core? & 0.5 & 0.49 & 0.59 & no? \\
\hline P/Whipple & & +0.5 & 0.36 & 0.40 & \\
\hline
\end{tabular}

a See: Sekanina (1971), Marsden and Sekanina (1971).

b Equals to $+0.8^{\mathrm{m}}$ before perihelion in 1959; and $+0.7^{\mathrm{m}}$ after perihelion in 1959 and in 1965 .

c Equals to $\pm 0.40^{\mathrm{m}}$ and $\pm 0.57^{\mathrm{m}}$, respectively, before perihelion in 1959 ; and to $\pm 0.34^{\mathrm{m}}$ and $\pm 0.40^{\mathrm{m}}$, respectively, after perihelion in 1959 and in 1965.

$\pm 0.26^{\mathrm{m}}$. This may suggest that a difference of $0.1^{\mathrm{m}}$ in the $H_{0}$ dispersion between the two phase laws might already be a meaningful discrimination level, if the dispersion itself is within, say, $\pm 0.5^{\mathrm{m}}$. To obtain more convincing results precise photometry must be applied.

\section{FINAL REMARKS}

We conclude that the hypothesis of fairly frequent collisions of interplanetary boulders with cometary nuclei, suggested in Paper 1 and examined from specific viewpoints in the present paper, looks reasonably consistent with the limited information available on the character and rate of disturbances observed in the motions of the 'erratic' short-period comets. Precise photometry of cometary nuclei, if conducted in the future, is believed to improve significantly our knowledge of the amount of mass and energy involved in the sort of collisions under consideration. We do not exclude a possibility of a different interpretation of the observed phenomena, but we do not see any at present that could compete with the impact hypothesis. We also feel that collisional processes involving fairly large objects of the solar system should be sul,jected to extensive investigations rather than rejected as ad hoc assumptions without seriously considering the chances, effects and characteristics of the collisional mechanism itself.

\section{ACKNOWLEDGMENTS}

The writer's thanks are due to B. G. Marsden for providing ephemerides of the comets and asteroids studied above, and to J. F. Veverka for discussing photometry problems.

\section{NOTE}

Since the time of the IAU Colloquium \#13 our understanding of the problems discussed in this paper has further advanced. We know of two more "erratic" comets, P/Finley and P/Comas Solá (Marsden, B. G., Sekanina, Z., and Yeomans, D. K., 1973 Comets and Nongravitational Forces. V. Astron. J. 78, 211-225). 


\section{REFERENCES}

Gehrels, T., Roemer, E., Taylor, R. C., and Zellner, B. H., 1970. Minor planets and related objects. IV. Asteroid (1566) Icarus, Astron. J., 75, 186-195.

HARwit, M., 1967. The cloud of interplanetary boulders, in The Zodiacal Light and the Interplanetary Medium, edited by J. L. Weinberg, NASA SP-150, Supt. of Documents, U.S. Govt. Printing Office, Washington, 307-313.

Marsden, B. G., 1969. Comets and nongravitational forces II, Astron. J., 74, 720-734.

- , 1970. Comets and nongravitational forces III, Astron. J., 75, 75-84.

- 1971 . The motion, splitting and present condition of Biela's comet, Bull. American Astron. Soc., 3, 269.

Marsden, B. G., and Sekanina, Z., 1971. Comets and nongravitational forces IV, Astron. J., 76, 1135-1151.

RAKos, K. D., 1960. Light variations of the fast moving minor planet discovered on October 22, 1960, by H. L. Giclas, Lowell Obs. Bull., 5, 28-29.

Rowmer, E., 1965. Observations of comets and minor planets, Astron. J., 70, 397-402.

- - 1967. Comet notes, Publ. Astron. Soc. Pacific, 79, 272-273, 372-374, 503-505, 632-634.

-C, 1968. Comet notes, Publ. Astron. Soc. Pacific, 80, 106-108, 345-347.

Rowmer, E., AND Lloyd, R. E., 1966. Observations of comets, minor planets, and satellites, Astron. J., 71, 443-457.

Roemer, E., Thomas, M., and Lloyd, R. E., 1966. Observations of comets, minor planets, and Jupiter VIII, Astron. J., 71, 592-601.

Serkanina, Z., 1968. Motion, splitting and photometry of Comet Wirtanen 1957 VI, Bull. Astron. Inst. Czech., 19, 153-170.

- , 1971. A core-mantle model for cometary nuclei and asteroids of possible cometary origin, in Physical Studies of Minor Planets, edited by T. Gehrels, NASA SP-267, Supt. of Documents, U.S. Govt. Printing Office, Washington, 423-428.

Veverka, J. F., 1970. Photometric and polarimetric studies of minor planets and satellites, PhD. dissertation, Harvard Univ., 97-107.

Yeomans, D. K., 1972. Nongravitational forces in comet Giacobini-Zinner, in The Motion, Evaluation of Orbits and Origins of Comets, edited by G. A. Chebotarev, E. I. KazimirchakPolonskaya and B. G. Marsden, D. Reidel Publ. Co., Dordrecht, Holland, 181-186. 